\title{
SIMPLE SHELL-MODEL EXPRESSIONS FOR COULOMB ENERGIES
}

\author{
K. T. HECHT \\ Physics Department, University of Michigan \\ Ann Arbor, Michigan ${ }^{\dagger}$
}

Received 27 February 1968

\begin{abstract}
It is shown that the main $n-T$ dependent terms of the Coulomb energy have the same general form in the Wigner supermultiplet scheme as in the low seniority limit of the $j-j$ coupling scheme of the shell model.
\end{abstract}

\section{Introduction}

As a result of the extensive work on isobaric analogue states, there has been renewed interest in the study of Coulomb displacement energies. Despite the simplicity of the Coulomb interaction, some of the recent analyses ${ }^{1,2}$ ) have been based on the Coulomb energy formula of Carlson and Talmi ${ }^{3,4}$ ) derived for proton configurations and applied to configurations of both protons and neutrons under the assumption that the seniority of the protons is a good quantum number (rather than isospin). This approach has been quite successful since the Coulomb energy shows only a weak dependence on total nucleon number and isospin. Although the $n-T$ dependence is weak, the accuracy of recent measurements of Coulomb displacement energies is such that the $n-T$ dependent terms should be taken into account properly in the theoretical treatment. Moreover, they are the source of the interesting odd-even and oscillatory $A$-dependent effects which have been observed experimentally ${ }^{5}$ ). Theoretical expressions for the $n-T$ dependent factors of the Coulomb energy have recently been derived for states of low seniority $(v \leqq 2)$ and simple configurations of the shell model in $j-j$ coupling ${ }^{6,7}$ ). Despite the simplicity of the assumptions, these expressions seem to be in remarkably good agreement with the experimental facts ${ }^{8}$ ). This is surprising since the seniority scheme may be a very poor approximation in nuclei where both neutrons and protons are filling the same shell. It may therefore be interesting to examine the nature of the $n-T$ dependent factors of the Coulomb energy for another limiting coupling scheme. The results of such a calculation are presented in this note for the Wigner supermultiplet scheme of a major oscillator shell. It is shown that the main $n-T$ dependent factors of the Coulomb energy have a form identical with that found in the seniority scheme in states in which the Wigner supermultiplet quantum numbers, total spin $S$ and isospin $T$ are assumed to be good quantum numbers.

+ Work supported in part by U.S. Office of Naval Research, Contract 1224 (59). 


\section{Results and discussion}

The Coulomb interaction between nucleons, when classified according to its irreducible tensor character in isospin space, can be written as

$$
V_{\mathrm{C}}=\sum_{i<j} \frac{e^{2}}{r_{i j}}\left\{\frac{1}{3}\left(\frac{3}{4}+\boldsymbol{t}_{i} \cdot \boldsymbol{t}_{j}\right)-\frac{1}{2}\left(t_{z_{i}}+t_{z_{j}}\right)+\frac{1}{3}\left(3 t_{z_{i}} t_{z_{j}}-\boldsymbol{t}_{i} \cdot \boldsymbol{t}_{j}\right)\right\}
$$

In first-order perturbation theory, this leads to the three terms of the Coulomb energy formula

$$
E=E^{(0)}-T_{z} E^{(1)}+\left[3 T_{z}^{2}-T(T+1)\right] E^{(2)} .
$$

The present interest is in the $n-T$ dependent terms of the isovector and isotensor coefficients $E^{(1)}$ and $E^{(2)}$. Results for the simplest coupling schemes are shown in table 1. For states of low seniority $v$ and reduced isospin $t$ for a simple configuration $j^{n}$, these results have been derived in refs. ${ }^{6,7}$ ). For states with $v=0$ and 1 , the Coulomb energy coefficients are given in terms of the two-body matrix elements

$$
V_{J}=\left\langle j^{2} J\left|\frac{e^{2}}{3 r_{i j}}\right| j^{2} J\right\rangle
$$

by the two coefficients

$$
b=\frac{2(j+1) \bar{V}_{2}-V_{0}}{2(2 j+1)}, \quad c=\frac{V_{0}-\bar{V}_{2}}{4(2 j+1)},
$$

where $\bar{V}_{2}$ is the average seniority-two matrix element ${ }^{4,6}$ ). The contributions from the interaction of the nucleons in the configuration $j^{n}$ with the core are given by

$$
a_{\mathrm{c}}=\sum_{J, j_{\mathrm{c}}} \frac{(2 J+1)}{(2 j+1)}\left\langle\left(j j_{\mathrm{c}}\right) J\left|\frac{e^{2}}{3 r_{i j}}\right|\left(j j_{\mathrm{c}}\right) J\right\rangle,
$$

in which $\left|\left(j j_{\mathrm{c}}\right) J\right\rangle$ are antisymmetrized states. Exact results for states with $v=2$ are given in ref. ${ }^{7}$ ). Although the form of the $n$ - $T$ dependent factors is similar, the coefficients ${ }^{\dagger}$ are more complicated functions of the two-body matrix elements $V_{J}$ and are dependent on the specific angular momentum $J_{2}$ of the $v=2$ state. Actual calculations, however, show that the dependence on $J_{2}$ is very weak. In the $1 \mathrm{f}_{\frac{7}{2}}$ shell, for example, Coulomb energies for states with different values of $J_{2} \operatorname{differ}^{8}$ ) from the average

+ The coefficient $f$ in eq. (90) of ref. ") is in error. The second of the two terms for $f$ should be replaced by

$$
\frac{2}{(2 j+1)}\left\{j \vec{V}_{\text {even }}-j\left(\overline{\left.V_{\text {even }} U_{J_{2}}\right)}-V_{J_{2}}+V_{0}\right\}\right.
$$

For $j \leqq \frac{7}{2}$, many of the coefficients of eq. (90), ref. ${ }^{7}$ ), can be simplified by the relation

$$
2\left(2 j \bar{V}_{\text {even }}-V_{0}\right)=(2 j-1)\left\{V_{J_{2}}-2 j\left(V_{\text {even }} U_{J_{2}}\right)\right\}
$$

valid only for $j \leqq \frac{7}{2} ; J_{2} \geqq 2$, even. 
K. T. HECHT

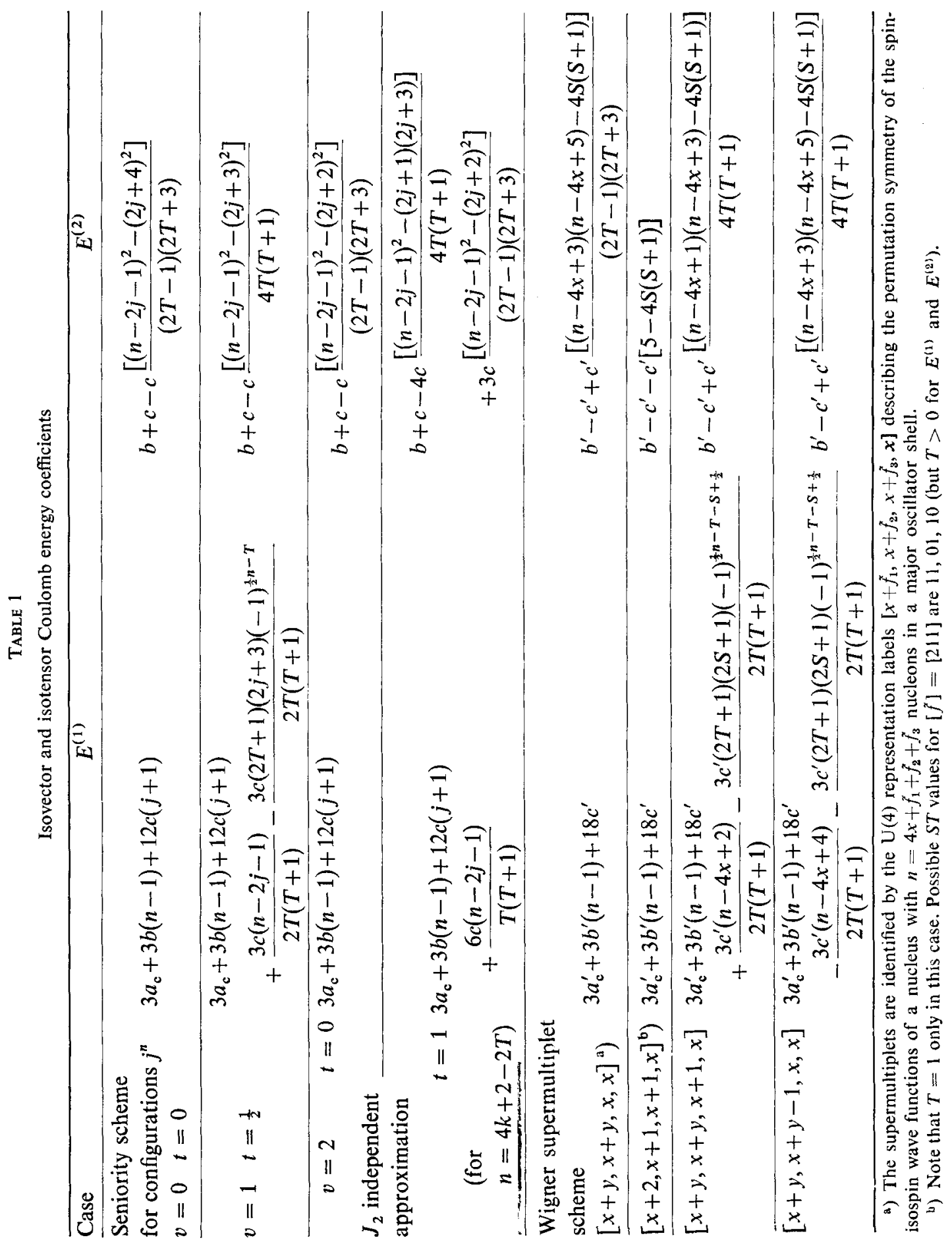


$v=2$ value by about $10 \mathrm{keV}$. It is therefore a good approximation to set $V_{J_{2}}=\bar{V}_{2}$ for all $J_{2} \geqq 2$, even. In this $J_{2}$-independent approximation, the Coulomb energies for $v=2$ states are again given in terms of the two coefficients $b$ and $c$ of eq. (3), and the $n-T$ dependent factors are only slightly more complicated than those for $v=0$. The results are shown in table 1.

Since the Coulomb energies are very insensitive functions of the spatial quantum numbers in the $j-j$ coupling scheme, a similar approximation can be expected to be good in other coupling schemes. In particular, by making such an approximation it is possible to calculate general algebraic expressions for the Coulomb energy in the Wigner supermultiplet scheme, exhibiting its dependence on $n, T, S$ and the Wigner supermultiplet quantum numbers [ $f$ ] [the notation is that of ref. $\left.{ }^{9}\right)$ ]. The derivations are based on recent calculations of SU(4) Wigner and Racah coefficients ${ }^{10,11}$ ) in general algebraic form. Such coefficients have been calculated for those irreducible representations of SU(4) for which the quantum numbers $S$ and $T$ are sufficient to specify completely the states of a given representation. Although this does not include all Wigner supermultiplets, it does include most of the cases of practical interest. In particular, the needed coefficients have been calculated for the SU(4) representations $[\tilde{f}]=[y y],[y y 1],[y, y-1]$, corresponding to spin-isospin functions whose symmetry is described by the partition numbers $[x+y, x+y, x, x],[x+y, x+y, x+1, x]$, $[x+y, x+y-1, x, x]$, where $x$ and $y$ are arbitrary integers. In the representation $\left[x+f_{1}, x+f_{2}, x+f_{3}, x\right]$ the numbers $x+f_{i}$ give the number of squares in the ith row of the Young tableau describing the permutation symmetry of the spin-isospin wave function of a nucleus with $n=4 x+f_{1}+f_{2}+f_{3}$ nucleons in a major oscillator shell. By including with the above the special SU(4) representation [211], all symmetries of actual importance for ground states of nuclei can be considered. Results for the four types of symmetries are shown in table 1 . The coefficients $b^{\prime}$ and $c^{\prime}$ can be calculated for each major oscillator shell. It is convenient to express them as

$$
b^{\prime}=\frac{1}{2}(\alpha+3 \beta), \quad c^{\prime}=\frac{1}{48}(\alpha-\beta) .
$$

Using harmonic oscillator wave functions, their values in units of $e^{2}[m \omega / 2 \pi \hbar]^{\frac{1}{2}}$ are given by

$$
\begin{array}{lll}
\text { for the 1p shell } & \alpha=\frac{19}{12}, & \beta=\frac{4}{3}, \\
\text { for the 2s, 1d shell } & \alpha=\frac{119}{96}, & \beta=\frac{7}{6} .
\end{array}
$$

The coefficients $b^{\prime}$ and $c^{\prime}$ have magnitudes similar to those of $b$ and $c$ for the corresponding subshells in $j-j$ coupling. The coefficient $a_{\mathrm{c}}^{\prime}$ is the orbital angular momentum analogue of eq. (4).

The $n-T$ dependence of the isovector and isotensor coefficients of the Coulomb energy is almost identical to that found in the seniority scheme. For even nuclei, $E^{(1)}$ shows a simple linear dependence on $n$. The additional terms found in odd nuclei and the $n-T$ dependent phase of these terms are the source of the odd-even and 
oscillatory $A$-dependent effects which are observed experimentally ${ }^{5}$ ). Since such terms are predicted for both the Wigner supermultiplet scheme and the low $v$ limit of the seniority scheme, it is reasonable to assume that they have a much more general validity.

\section{Derivation of the Wigner supermultiplet formulae}

For the derivation, it is convenient to express the Coulomb interaction in terms of the projection operators $Q_{n l}$ introduced by Brink ${ }^{12}$ ). The operators $Q_{n l}$ are twobody operators which project out those parts of a two-nucleon state with relative motion in the state $(n l)$ of a harmonic oscillator basis. In addition, the basic projection operators will be classified according to their irreducible tensor character under $\mathrm{SU}(4)$. It is convenient to define operators $Q_{n l,\left[\tilde{f}_{0}\right] S_{0} T_{0}}$ with $\mathrm{SU}(4)$ tensor character $\left[\tilde{f}_{0}\right]$ and spin, isospin character $S_{0}, T_{0}$. Since the Coulomb interaction is spin independent, $S_{0}=0$; while $T_{0}=0,1,2$ for the isoscalar, vector and tensor parts of eq. (1). Such operators can be expressed as

$$
\begin{aligned}
& Q_{n l,\left[\tilde{f}_{0}\right] 0 T_{0}}=\frac{1}{2} \sum_{\substack{\mathcal{N} \mathscr{L} L \\
n_{\alpha} l_{\alpha} n_{\beta} l_{\beta} l_{\gamma} n_{\delta} l_{\delta}}}\left\langle n l \mathscr{N} \mathscr{L}, L \mid n_{\alpha} l_{\alpha} n_{\beta} l_{\beta}, L\right\rangle\left\langle n l \mathscr{N} \mathscr{L}, L \mid n_{\gamma} l_{\gamma} n_{\delta} l_{\delta}, L\right\rangle \\
& \times\left[\frac{\operatorname{dim}\left[\tilde{f}_{0}\right]}{\operatorname{dim}[\tilde{f}]}\right]^{\frac{1}{2}} \sum_{S T M_{S} M_{T} M_{L}}\left\langle\left[\tilde{f}_{0}\right] 0 T_{0} ;[\tilde{f}] S T||[\tilde{f}] S T\right\rangle\left\langle T_{0} 0 T M_{T} \mid T M_{T}\right\rangle \\
& \times \mathscr{A}_{\alpha \beta, M_{L} M_{S} M_{T}}^{+[\tilde{f}] L S T} \mathscr{A}_{\gamma \delta, M_{L} M_{S} M_{T}}^{[\tilde{f}] L S T}
\end{aligned}
$$

The $n, l, \mathscr{N}, \mathscr{L}, \ldots$ dependent factors are Moshinsky-Brody brackets ${ }^{13}$ ). The harmonic oscillator quantum numbers $n, l, \mathscr{N}, \mathscr{L}$ describe the relative and center-ofmass motion of the two-nucleon states. The operators $\mathscr{A}^{\dagger}$ are pair creation operators defined in terms of single-particle creation operators by

$$
\begin{aligned}
\mathscr{A}_{\alpha \beta,}^{\dagger\left[\tilde{\beta} M_{L} M_{S} M_{T}\right.}= & \sum_{m_{l_{\alpha}} m_{s_{\alpha}} m_{t_{\alpha}}}\left\langle l_{\alpha} m_{l_{\alpha}} l_{\beta} m_{l_{\beta}} \mid L M_{L}\right\rangle\left\langle\frac{1}{2} m_{s_{\alpha}} \frac{1}{2} m_{s_{\beta}} \mid S M_{S}\right\rangle \\
& \times\left\langle\frac{1}{2} m_{t_{\alpha}} \frac{1}{2} m_{t_{\beta}} \mid T M_{T}\right\rangle a_{n_{\alpha} l_{\alpha} m_{l_{\alpha}} m_{s_{\alpha}} m_{t_{\alpha}}}^{\dagger} a_{n_{\beta} l_{\beta} m_{l_{\beta}} m_{s_{\beta}} m_{t_{\beta}}}^{\dagger} .
\end{aligned}
$$

The $S U(4)$ tensor character is $[\tilde{f}]=2$ for the $S T$ values 11 or 00 , possible for $2 n+l=$ odd integer. It is $[\tilde{f}]=[11]$ for the $S T$ values 01 or 10 , compatible with $2 n+l=$ even integer. The pair annihilation operators $\mathscr{A}$ are defined as the hermitean conjugates of $\mathscr{A}^{\dagger}$. Such operators have tensor character given by

$$
\mathscr{A}_{M_{L} M_{S} M_{T}}^{[\tilde{\tilde{I}} \mathrm{~L} L S T}=-(-1)^{L-M_{L}+\eta+S-M_{S}+T-M_{T}} T_{-M_{L},-M_{S},-M_{T}}^{\left[\tilde{\left.\tilde{f}^{*}\right] L S T}\right.},
$$

where $\left[\tilde{f}^{*}\right]$ is the $\mathrm{SU}(4)$ representation conjugate to $[\tilde{f}]$; that is, $\left[2^{*}\right]=[222]$, while

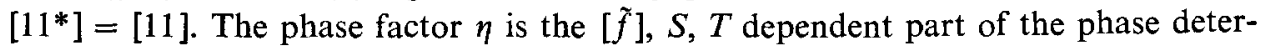
mined by the conjugation properties of the $\mathrm{SU}(4)$ states, which is given for most 
of the simple SU(4) representations in ref. ${ }^{11}$ ). From the Kronecker products

$$
\begin{aligned}
{[2] \times[222] } & =[0]+[211]+[422], \\
{[11] \times[11] } & =[0]+[211]+[22],
\end{aligned}
$$

it can be seen that the two-body operators include operators with SU(4) irreducible tensor character given by $\left[\tilde{f}_{0}\right]=[0],[211],[422]$ and [22]. In eq. (7), dim $[\tilde{f}]$ is the dimension of the SU(4) irreducible representation $[\tilde{f}]$, while the double-barred coefficient is a reduced SU(4) Wigner coefficient of the type calculated in refs. ${ }^{10,11}$ ). This double-barred coefficient together with the ordinary isospin Wigner coefficient (and a spin Wigner coefficient, if needed) makes up the full SU(4) Wigner coefficient needed for the coupling of two SU(4) irreducible representations. The operators $Q_{n l,\left[\tilde{f}_{0}\right] 0 T_{0}}$ can also be expressed in terms of isospin operators for nucleons $i$ and in the expansion of the two-body operators, as shown explicitly in table 2.

TABLE 2

Isospin-dependent factors of the operators $Q_{n l,\left[\tilde{f_{0}}\right] 0 T_{0}}$

\begin{tabular}{rllll}
\hline$\left[f_{0}\right]$ & $S_{0}$ & $T_{0}$ & $2 n+l$ & \multicolumn{1}{c}{$\varphi\left(\boldsymbol{t}_{i}, \boldsymbol{t}_{j}\right)$} \\
\hline$[0]$ & 0 & 0 & even, odd & 1 \\
{$[22]$} & 0 & 0 & even & $\frac{1}{2}\left(1+4 \boldsymbol{t}_{i} \cdot \boldsymbol{t}_{j}\right)$ \\
{$[422]$} & 0 & 0 & odd & $\frac{1}{6}\left(-3+20 \boldsymbol{t}_{i} \cdot \boldsymbol{t}_{j}\right)$ \\
{$[211]$} & 0 & 1 & even & $\sqrt{3}\left(t_{z_{i}}+t_{z_{j}}\right)$ \\
{$[211]$} & 0 & 1 & odd & $\sqrt{\frac{5}{3}}\left(t_{z_{i}}+t_{z_{j}}\right)$ \\
{$[22]$} & 0 & 2 & even & $-2\left(3 t_{z_{i}} t_{z_{j}}-\boldsymbol{t}_{i} \cdot \boldsymbol{t}_{j}\right)$ \\
{$[422]$} & 0 & 2 & odd & $2 \sqrt{\frac{5}{3}}\left(3 t_{z_{i}} t_{z_{j}}-\boldsymbol{t}_{i} \cdot \boldsymbol{t}_{j}\right)$
\end{tabular}

$Q_{n l,\left[\tilde{f}_{0}\right] 0 T_{0}}=\sum_{i<j}\left(q_{n l}\right)_{i j} \varphi\left(t_{i}, t_{j}\right)$.

In terms of the operators $Q_{n l,\left[\tilde{f}_{0}\right] 0 T_{0}}$, the Coulomb interaction can be expressed as

$$
\begin{aligned}
V_{\mathrm{C}}= & \sum_{n l(2 n+l=\mathrm{even})}\langle n l\|V\| n l\rangle\left\{\frac { 1 } { \sqrt { } 6 } \left[Q_{n l,[0] 00}+Q_{n l,[22] 00]}\right.\right. \\
& \left.-\frac{1}{\sqrt{2}} Q_{n l,[211] 01}-\frac{1}{\sqrt{6}} Q_{n l,[22] 02}\right\} \\
+ & \\
+\sum_{n l(2 n+l=\text { odd })}\langle n l\|V\| n l\rangle\left\{\frac{1}{\sqrt{ } 10}\left[3 Q_{n l,[0] 00}+Q_{n l,[422] 00}\right]\right. & \left.-\sqrt{\frac{3}{2}} Q_{n l,[211] 01}+\frac{1}{\sqrt{2}} Q_{n l,[422] 02}\right\},
\end{aligned}
$$

where the notation for the double-barred matrix elements is that of ref. ${ }^{13}$ ). To identify the irreducible tensor character of the spatial part of the interaction in each major 
harmonic oscillator shell, it is convenient to group the $Q_{n l}$ in the following way: for the $1 \mathrm{p}$ shell

$$
\begin{array}{rlrl}
\frac{1}{e^{2}}\left[\frac{2 \pi \hbar}{m \omega}\right]^{\frac{1}{2}} \sum_{n l}\left\langlen l \left\||V \| n l\rangle Q_{n l}\right.\right. & \\
= & \frac{19}{12}\left\{Q_{00}+\left(Q_{10}+Q_{02}\right)\right\}+\frac{4}{3}\left\{Q_{01}\right\} & & \text { SU(3) scalars } \\
& +\frac{1}{10}\left\{\left(5 Q_{10}-Q_{02}\right)\right\} & & \text { SU(3) (22) tensor, }
\end{array}
$$

for the $2 \mathrm{~s}, 1 \mathrm{~d}$ shell

$$
\begin{aligned}
& \frac{1}{e^{2}}\left[\frac{2 \pi \hbar}{m \omega}\right]^{\frac{1}{2}} \sum_{n l}\langle n l \||V| \mid n l\rangle Q_{n l} \\
& =\frac{119}{96}\left\{Q_{00}+\left(Q_{10}+Q_{02}\right)+\left(Q_{20}+Q_{12}+Q_{04}\right)\right\}+\frac{7}{6}\left\{Q_{01}+\left(Q_{11}+Q_{03}\right)\right\} \quad \mathrm{SU}(6) \text { scalars } \\
& +\frac{7}{96}\left\{3 Q_{00}+3\left(Q_{20}+Q_{12}+Q_{04}\right)-\left(Q_{10}+Q_{02}\right)\right\} \quad \mathrm{SU}(3) \text { scalar, SU(6)[42222] tensor } \\
& +\frac{1}{72}\left\{\left(21 Q_{20}-6 Q_{12}+Q_{04}\right)\right\} \\
& \mathrm{SU}(3)(44) \text { tensors } \\
& +\frac{11}{280}\left\{\left(7 Q_{20}+4 Q_{12}-3 Q_{04}\right)\right\} \\
& +\frac{1}{10}\left\{\left(5 Q_{10}-Q_{02}\right)\right\}+\frac{1}{35}\left\{\left(7 Q_{11}-3 Q_{03}\right)\right\} \quad \text { SU (3) }(22) \text { tensors. }
\end{aligned}
$$

For each shell only the first two terms (in curly brackets) are completely invariant under all the symmetry transformations of the symmetry groups associated with the space part of the wave functions, ("full space scalars"). These are given by the operators $\sum Q_{n l}$ summed over all possible values of $n l$ with $2 n+l=$ even integer or odd integer, respectively. They could also be identified with the operators $n_{+}$and $n_{-}$, where $n_{+}$(or $n_{-}$) counts the number of spatially symmetric (or antisymmetric) nucleon pairs within a major shell ${ }^{12}$ ). The coefficients of $n_{+}$and $n_{-}$are the constants $\alpha$ and $\beta$ of eq. (6). The remaining terms have the SU(3) tensor character ${ }^{14}$ ) indicated by the quantum numbers $(\lambda \mu)$. In the $2 \mathrm{~s}, 1 \mathrm{~d}$ shell there is one term which is an $\mathrm{SU}(3)$ scalar but has $\mathrm{SU}(6)$ tensor character [42222], the third term of eq. (13). Its eigenvalue ${ }^{12}$ ) is given by $\frac{2}{3}\left\{\frac{1}{2} n(n-6)+\frac{9}{4} G\right\}$, where $G$ is the $\mathrm{SU}(3)$ Casimir invariant. The various terms enclosed by curly brackets have matrix elements of comparable orders of magnitude. It can thus be seen from the coefficients of eqs. (12) and (13) that only the full space scalars make a significant contribution to the Coulomb energy. The remaining terms are smaller by an order of magnitude, and to a good approximation they can be neglected. The full space scalars have matrix elements which can depend only on nucleon number $n$ and the symmetry quantum numbers [f] and through their SU(4) tensor character as exhibited in eq. (11) on the spin and isospin of a state. In the approximation in which only the full space scalars are retained, Coulomb energy matrix elements can be calculated in general algebraic form. The matrix element of a full space scalar can be expressed in the usual fractional parentage format ${ }^{9}$ ), with only the spin-isospin part of the c.f.p. needed ${ }^{9}$ ). In performing the fractional 
parentage sums, it is advantageous to recognize the relationship ${ }^{11,15}$ ) between the $n$ to $n-2$ particle spin-isospin c.f.p. and the SU(4) Wigner coefficients needed for the coupling of SU(4) irreducible representations $\left[\tilde{f}_{n-2}\right]$ with $\left[\tilde{f}_{2}\right]$ to resultant $\left[\tilde{f}_{n}\right]$. By exploiting this relationship, sums over spin and isospin quantum numbers can be performed and the matrix elements can be expressed in terms of resultant SU(4) Wigner and Racah coefficients. The matrix element of a full space scalar can be written as

$$
\begin{aligned}
\left\langle\left[\tilde{f}_{n}\right] S M_{S} T M_{T}\left|\sum_{n l} Q_{n l,\left[\tilde{f}_{0}\right] 0 T_{0}}\right|\left[\tilde{f}_{n}\right] S M_{S} T M_{T}\right\rangle \\
=\frac{1}{2}[n(n-1)]_{\left[\tilde{f}_{n-2}\right]\left[\tilde{f}_{2}\right]} \frac{\mathscr{N}\left(\left[\tilde{f}_{n-2}\right]\right)}{\mathscr{N}\left(\left[\tilde{f}_{n}\right]\right)} \frac{\left\langle\left[\tilde{f}_{2}\right] \| Q_{\left[\tilde{f}_{0}\right]}||\left[\tilde{f}_{2}\right]\right\rangle}{\left[\operatorname{dim}\left[\tilde{f}_{0}\right]\right]^{\frac{1}{2}}} \\
\quad \times \sum_{\rho}(-1)^{\eta_{0}+T_{0}}\left\langle\left[\tilde{f}_{n}\right] S T ;\left[\tilde{f}_{0}\right] 0 T_{0}||\left[\tilde{f}_{n}\right] S T\right\rangle_{\rho}\left\langle T M_{T} T_{0} 0 \mid T M_{T}\right\rangle \\
\quad \times \frac{U\left\{\left[\tilde{f}_{n}\right]\left[\tilde{f}_{2}^{*}\right]\left[\tilde{f}_{n}\right]\left[\tilde{f}_{2}\right] ;\left[\tilde{f}_{n-2}\right]\left[\tilde{f}_{0}\right]\right\}_{\rho}}{U\left\{\left[\tilde{f}_{n}\right]\left[\tilde{f}_{2}^{*}\right]\left[\tilde{f}_{n}\right]\left[\tilde{f}_{2}\right] ;\left[\tilde{f}_{n-2}\right][0]\right\}} .
\end{aligned}
$$

Here $n$ is the number of nucleons within a major oscillator shell; $\mathscr{N}\left(\left[\tilde{f}_{n}\right]\right)$ is the dimension of the irreducible representation $\left[\tilde{f}_{n}\right]$ of the symmetric group giving the permutation symmetry of the full $n$-nucleon state ${ }^{9}$ ). The $Q$-operators of eq. (7) have been normalized such that their reduced matrix elements are given solely by the dimensions of the irreducible representations of SU(4)

$$
\left\langle\left[\tilde{f}_{2}\right]\left\|Q_{\left[\tilde{f}_{0}\right]}\right\|\left[\tilde{f}_{2}\right]\right\rangle=\left[\frac{\operatorname{dim}\left[\tilde{f}_{0}\right]}{\operatorname{dim}\left[\tilde{f}_{2}\right]}\right]^{\frac{1}{2}}
$$

The phase factor $\eta$ is defined in eq. (9). The $S, T$ dependent double-barred coefficient is a reduced SU(4) Wigner coefficient. The $U$-coefficients are SU(4) Racah coefficients in unitary form. [The notation is a straightforward generalization of that for the angular momentum $U$-coefficient ${ }^{9}$ ).] The quantum numbers $\rho$ are needed in those cases in which the products $\left[\tilde{f}_{n}\right] \times\left[\tilde{f}_{0}\right]$ are not simply reducible but contain the representation $\left[\tilde{f}_{n}\right]$ more than once. Such cases occur for the representations $\left[\tilde{f}_{0}\right]=$ [211] and [422]. The $U$-coefficient containing the scalar representation [0] is a convenient way of expressing dimensional and phase factors. By expressing the full matrix element in the form of a ratio of two $\mathrm{SU}(4) U$-coefficients, the result is independent of particular phase conventions used in the calculation of the $\mathrm{SU}(4)$ Wigner coefficients. The SU(4) Wigner and Racah coefficients needed for the irreducible representations of table 1 have been calculated as functions of $y, S$ and $T$ in ref. ${ }^{11}$ ). For these representations, the matrix element (14) can be evaluated in general algebraic form. The $S, T$ dependence arises solely through the SU(4) Wigner coefficient of eq. (14). The results for the isovector and tensor coefficients of the 
Coulomb energy are shown in table $1^{\dagger}$. The isoscalar coefficient for all four types of $\mathrm{SU}(4)$ symmetries has the value

$$
\begin{aligned}
E^{(0)}= & \frac{1}{6} \alpha\left(n_{+}\right)+\frac{3 \beta}{10}\left(n_{-}\right)-\frac{1}{30} \beta C_{\mathrm{SU}(4)} \\
& +\frac{1}{12}(\alpha+\beta) T(T+1)-\frac{1}{12}(\alpha-\beta) S(S+1),
\end{aligned}
$$

where $n_{+}\left(n_{-}\right)$is the number of spatially symmetric (antisymmetric) nucleon pairs in the representation $\left[\tilde{f}_{n}\right]$ and $C_{\mathrm{SU}(4)}$ the eigenvalue of the $\mathrm{SU}(4)$ Casimir operator.

Further applications of eq. (14) are given in ref. ${ }^{11}$ ).

It is a pleasure to acknowledge many valuable and stimulating discussions with J. Jänecke.

† Since the magnitude of the $n-T$ dependent terms is determined entirely by the coefficient $c^{\prime}$, it should be pointed out that this is of the same order of magnitude as the coefficients of the neglected SU(6) and SU(3) tensor terms of eqs. (12) and (13). These tensor terms, however, cannot change the nature of the $y, S, T$ dependent factors for the Coulomb energy. Even if these tensor terms cannot be neglected completely, the Wigner supermultiplet expressions of table 1 are exact if applied to the average Coulomb energies for all states of an $S U(3)$ or $S U(6)$ multiplet in the $1 \mathrm{p}$ or $2 \mathrm{~s}, 1 \mathrm{~d}$ shell, respectively.

\section{References}

1) M. Harchol, A. A. Jaffe, J. Miron, I. Unna and J. Zioni, Nucl. Phys. A90 (1967) 459

2) R. Sherr, Phys. Lett. 24B (1967) 321

3) B. C. Carlson and I. Talmi, Phys. Rev. 96 (1954) 436

4) A. de-Shalit and I. Talmi, Nuclear shell theory (Academic Press, New York, 1963)

5) J. Jänecke, in Isobaric spin in nuclear physics, ed. by J. D. Fox and D. Robson (Academic Press, New York, 1966) p. 60

6) K. T. Hecht, ibid, p. 823

7) K. T. Hecht, Nucl. Phys. A102 (1967) 11

8) J. Jänecke, Nucl. Phys. A114 (1968) 433

9) H. A. Jahn and H. Van Wieringen, Proc. Roy. Soc. A209 (1951) 502

10) S. C. Pang, dissertation, University of Michigan (1967)

11) S. C. Pang and K. T. Hecht, to be published

12) D. M. Brink, Nucl. Phys. 40 (1963) 593

13) T. A. Brody and M. Moshinsky, Tables of transformation brackets for nuclear shell-model calculations (Monografias del Instituto de Fisica, Mexico, 1960)

14) G. Neely, dissertation, University of Michigan, to be published

15) V. I. Kukulin, Y. F. Smirnov and L. Majling, Nucl. Phys. A103 (1967) 681 